\title{
Maternal Exposure to Synthetic Chemicals and Obesity in the Offspring: Recent Findings
}

\author{
Yun Liu ${ }^{1}$ • Karen E. Peterson ${ }^{1,2,3}$
}

Published online: 24 September 2015

(C) Springer International Publishing AG 2015

\begin{abstract}
Experimental studies suggest perinatal exposures to synthetic chemicals may be associated with early onset obesity, although this hypothesis has not been extensively examined in humans. This article summarizes the evidence relating maternal perinatal exposure to common persistent organic compounds (polychlorinated biphenyl, dichlorodiphenyldichloroethylene, dichlorodiphenyltrichloroethane, hexachlorobenzene, hexachlorocyclohexane), perfluoroalkyls, perfluorooctane sulfonate, polybrominated diphenyl ethers and tributyltin, and nonpersistent compounds (phthalates, bisphenol A) on child obesity during sensitive developmental periods. Twenty-two epidemiologic studies published from 2011 to 2015 offer inconsistent support for the obesogenic effects of most substances and are limited by relatively small sample sizes and indirect measures of adiposity. The clearest findings suggest an influence of maternal dichlorodiphenyldichloroethylene exposure on offspring overweight and obesity. Recommendations for future epidemiological research include longer follow-up of effects of pre- and postnatal exposures in large samples; utilization of
\end{abstract}

This article is part of the Topical Collection on Synthetic Chemicals and Health

Karen E. Peterson

karenep@umich.edu

Yun Liu

yunliu@umich.edu

1 Department of Nutritional Sciences, University of Michigan School of Public Health, 1415 Washington Heights, 1-1867, Ann Arbor, MI 48109-2029, USA

2 Center for Human Growth and Development, University of Michigan, Ann Arbor, MI, USA

3 Departments of Nutrition and of Health and Social Behavior, Harvard W.T. Chan School of Public Health, Boston, MA, USA direct measures of adiposity; and consideration of effect modification by sex, birth weight, dietary fat, and maternal weight status.

Keywords Environmental obesogen · Maternal exposure · Infant growth $\cdot$ Weight gain $\cdot$ Overweight $\cdot$ Obesity $\cdot$ Child

\section{Introduction}

In recent decades, childhood obesity has emerged as a global public health crisis. The number of infants and preschool children who were overweight and obese worldwide rose from 32 million in 1990 to 42 million in 2013, and this total is estimated to increase to 70 million by 2025 [1]. In the USA, the prevalence of obesity in children increased from $7 \%$ in 1980 to $18 \%$ in 2012 and the proportion of obese adolescents increased from 5 to $21 \%$ over the same period $[2,3]$. Earlyonset obesity can lead to a number of major health conditions. In the short term, obese children are more likely to have a higher number of risk factors for cardiovascular disease [4] and to manifest prediabetes [5], fatty liver disease, musculoskeletal disease, breathing problems as well as social and psychological disorders [6-8]. Childhood obesity increases the likelihood of being obese as an adult [9] and is associated with greater morbidity and mortality in early adulthood [10-12] as well as risk of heart disease, type 2 diabetes, stroke, osteoarthritis and several types of cancer during later adulthood [13]. Obesity has surpassed cigarette smoking as the first preventable cause of death in the USA, imposing an enormous economic burden $[14,15]$. Dramatic obesity trends coupled with challenges of short- and long-term management of this chronic condition [16] and highlight the importance of disentangling the origins of pediatric obesity as a basis for mounting effective prevention strategies. 
Obesity trends are widely attributed to changes in diet, a sedentary lifestyle and genetic predisposition, but these risk factors do not fully account for the genesis and pattern of the obesity epidemic $[17,18]$. Since the late nineteenth century, the environment to which humans are exposed has changed due to the exponential growth in the production and use of synthetic chemicals [19]. In 2002, researchers proposed that endocrine disrupting chemicals (EDCs) may contribute to increases in obesity, offering ecologic evidence that elevated production of synthetic substances that coincided with US obesity trends [17]. Associations between high doses of EDCs and weight loss as well as low doses of the same chemicals and weight gain have been reported [17]. EDCs promoting weight gain and obesity collectively have been termed "obesogens" [20]. Scientific findings suggest these EDCs may cause obesity by interfering with lipid metabolism to stimulate adipogenesis and promote fat storage, shifting the metabolic set points to favor positive energy balance or altering hormonal control of appetite and satiety [20-22]. Compelling evidence suggests obesity is programmed as early in life as the intrauterine period [23, 24]. The environmental obesogen hypothesis [18] underlies the notion that fetal exposure to certain EDCs make affected persons susceptible to the induction of fat mass and excessive weight by modifying the epigenome of multipotent stromal stem cells, favoring them to the adipocyte lineage at the cost of bone. Thus, intrauterine exposure to obesogens may alter compartment of stem cells and predispose exposed individuals for adipogenic endpoints $[24,25]$. Nevertheless, compared with relatively extensive animal studies, epidemiological research investigating the association of maternal toxicants with children's postnatal obesity is limited.

This review considers the recent evidence examining impacts of maternal exposure to synthetic compounds on obesity and related measures of fat mass and distribution in human offspring. We focus on epidemiologic studies published over a 5-year period from 2011 to 2015, given the availability of three extensive reviews of earlier studies [26-28], summarized below.

Prior to 2011, numerous experimental reports and a limited number of epidemiological studies linked developmental exposure to certain classes of synthetic chemicals to postnatal obesity. Animal studies suggested that low levels of polychlorinated biphenyl (PCBs) may promote adipocyte differentiation, peroxisome proliferator-activated receptor (PPAR) $\gamma$ expression, and weight gain in offspring [29], but most prospective studies published before 2011 found no association to support this mechanism in humans [26]. Several organochlorine pesticides have been demonstrated to play a role in obesity. Earlier prospective human studies document an association between elevated levels of dichlorodiphenyltrichloroethane (DDT) or its main metabolite dichlorodiphenyldichloroethylene (DDE) during pregnancy and postnatal obesity in offspring [26]. The few cohort studies that examined the obesogenic effects of other organochlorine pesticides such as hexachlorobenzene (HCB) and hexachlorocyclohexane $(\mathrm{HCH})$ provided inconsistent conclusions, whereas animal studies did not support such a hypothesis [26]. Far fewer researchers have examined the obesogenicity of other persistent organic pollutants, including perfluorinated compounds (PFCs), polybrominated diphenyl ethers (PBDE), or oganotins. Nonpersistent compounds such as phthalates and bisphenol A (BPA) have been related to offspring obesity in experimental studies, but human data are insufficient to support such a relationship [27]. Although organophosphates potentially affect lipid metabolism $[28,30,31]$, no available evidence supports their obesogenicity.

We identified 22 human reports published between 2011 and 2015 that examined the relationship of perinatal exposure to (1) persistent organic pollutants including PCBs, DDE, DDT, HCB, HCH, perfluroalkyls (PFOA), perfluorooctane sulfonate (PFOS), polybrominated diphenyl ethers (PBDE), and tributyltin (TBT) or (2) nonpersistent compounds such as phthalates and BPA with obesity-related outcomes in offspring. After consultation with a research librarian, we searched PubMed using keywords including "Environmental obesogen," "PCBs, DDE, DDT, HCB, HCH, PFOA, PFOS, PBDE and TBT," "maternal exposure," "infant growth," "weight gain, overweight, obesity." We restricted our review to human, nonexperimental studies with a prospective longitudinal design that examined the associations of maternal perinatal exposures to obesity-related outcomes in their offspring. Most reports considered the influence of POPs, particularly DDE and PCBs, while fewer investigated the impact of other compounds. Studies involved different lengths of follow-up that provide insights on toxicant effects during subsequent sensitive periods for obesity development [7], although few followed children into adolescence or early adulthood. Across the 22 studies, researchers primarily relied on measured anthropometry for obesity-related outcomes including weight gain, body mass index (BMI), waist circumference (WC), and waist-hip ratio (WHR), while only two studies utilized direct measures of adiposity (Table 1).

\section{Persistent Organic Pollutants}

Persistent organic pollutants (POPs) are known EDCs and a group of synthetic chemicals that have been widely used as pesticides, solvents, pharmaceuticals, or industrial chemicals [32]. These chemicals have raised global concern due to their potential for long-range transport, capacity to persist in the environment, ability to bioaccumulate in ecosystems, as well as their marked negative effects on human health [33]. Humans are exposed to these persistent substances primarily through dietary ingestion, inhalation, or dermal exposure [34]. Although POPs are presently banned or restricted through the 


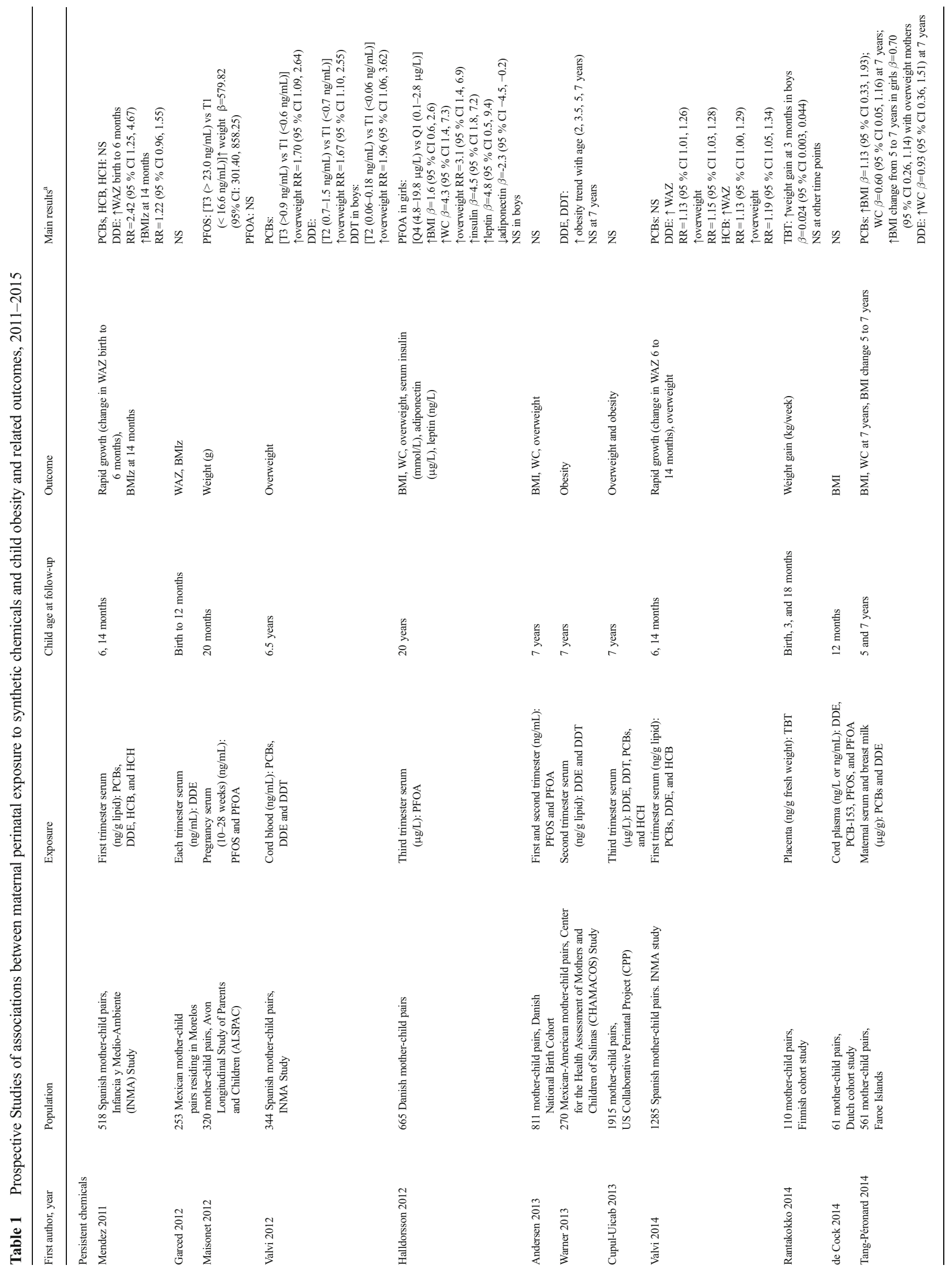




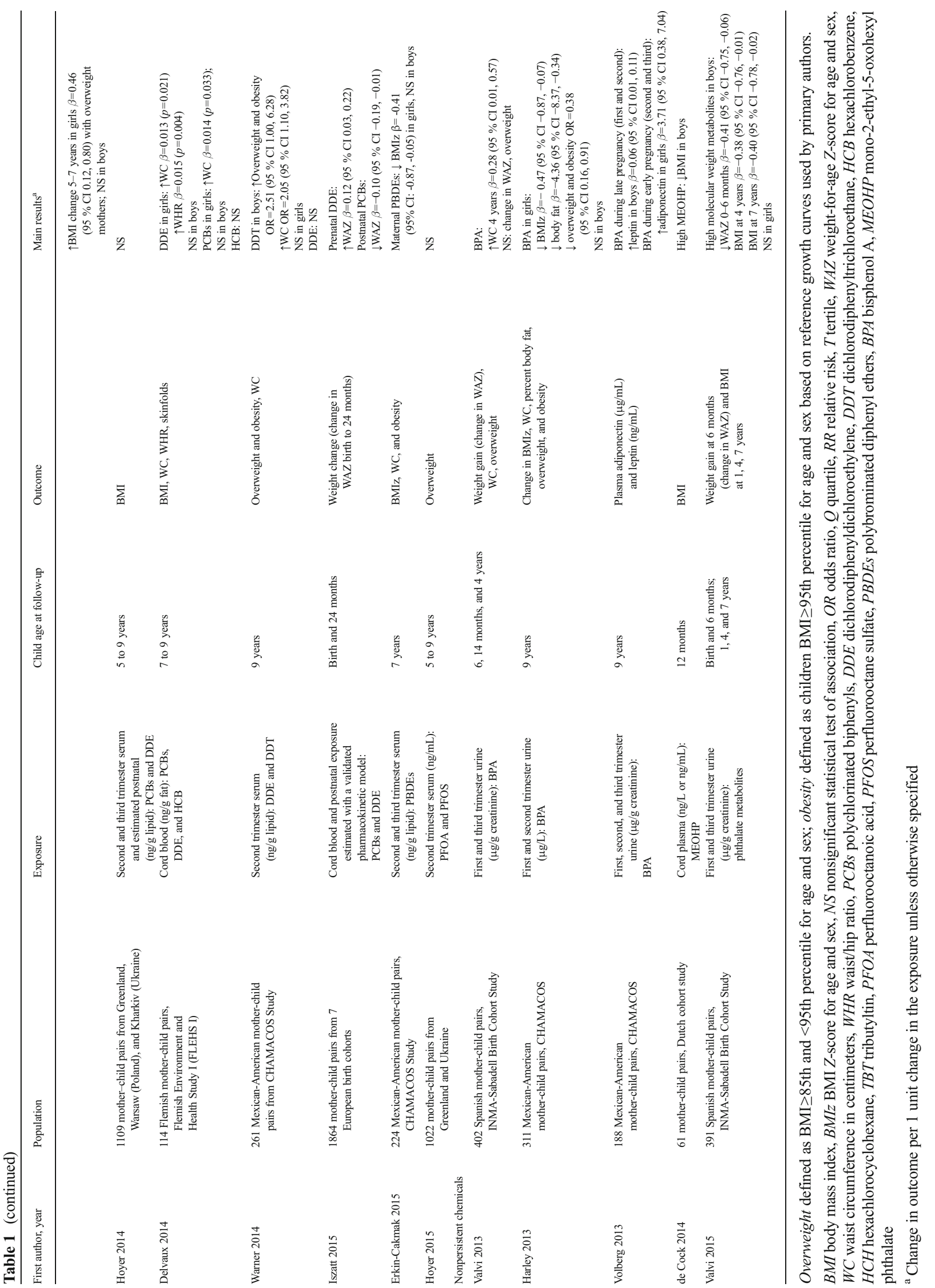


Stockholm Convention treaty, these toxicants can bioaccumulate within the food chain and are still detectable in human tissues around the world [27]. For instance, certain persistent chemicals can be detected at measurable levels in pregnant women. Specifically, PCBs, organochlorine pesticides, PFCs, and PBDEs were found in $99-100 \%$ of pregnant women [35]. Additionally, several POPs have been measured in cord blood, placental, and amniotic fluid as well as in human breast milk [36-38]. Therefore, it is possible that perinatal POPs exposure may start in utero by passing through the placenta and continue after delivery through breastfeeding. POPs are categorized into dioxins/dioxin-like substances or non-dioxin-like substances, as determined by their capacity to bind the aryl hydrocarbon receptor (AhR) [39]. Dioxins and dioxin-like compounds such as coplanar PCBs can promote adipogenesis by increasing PPAR expression [40] and by disturbing initiation of estrogen receptors to encourage the development of obesity [41]. In contrast, the underlying mechanism is not fully understood for non-dioxin-like compounds such as non-coplanar PCBs, organochlorine pesticides, $\mathrm{HCB}, \mathrm{HCH}, \mathrm{PFCs}$, and PBDE. Previous studies [27] suggest that DDT and $\mathrm{p}, \mathrm{p}^{\prime}$-DDE can exert toxicity through antiandrogenic, estrogenic, and antiestrogenic effects. HCB may lead to disrupted gluconeogenic reactions. PFOAs and TBT can affect fat storage, adipocyte differentiation, and insulin sensitivity via interfering with PPAR expression [27].

\section{PCBs}

Eighteen of the 22 prospective studies between 2011 and 2015 we reviewed evaluated the associations between maternal exposures to POPs and the development of obesity in offspring. Most of the prospective studies we reviewed failed to support the hypothesis that $\mathrm{PCBs}$ during pregnancy predict the risk of infant or child obesity prior to adolescence (Table 1). In the Spanish Infancia y Medio-Ambiente (INMA)-Sabadell birth cohort, serum PCB levels during the first trimester were not associated with rapid weight gain in the first 6 months of life or subsequent overweight at 14 months, although this study was limited by sample size [ $n=518]$ [42 $\bullet$. An expansion of the original INMA-Sabadell cohort to a larger study population $[n=1285]$ confirmed earlier findings that PCBs were unrelated to infant growth in this cohort [43]. A Dutch cohort study also found no association between PCB-153 measured in cord blood and infant growth [44-]. Two studies found no evidence for effects of PCBs on weight status in school-age children. A prospective cohort study [45] of maternal-child pairs from Greenland, Poland, and Ukraine found no clear associations between pregnancy PCB and the children's BMIs at 5 to 9 years. This study was consistent with earlier findings [46] from the US Collaborative Perinatal Project (CPP) showing that high levels of total PCB exposures during the third trimester were not linked to overweight or obesity in children aged 7 years. Notably, PCB exposure levels were relatively high in this population since blood samples were collected before these toxic substances were phased out in the USA.

Counter to the studies above that failed to demonstrate a relationship of PCBs to obesity-related outcomes in infancy or at school entry, three reports provide evidence that prenatal PCB exposure may affect growth rate, BMI, and measures of fat distribution and suggest effects on different anthropometric outcomes may vary in direction, as well as by age and sex (Table 1). In a Flemish cohort [47], PCB levels in cord blood were positively associated with the WC, an indirect measure of central fat distribution in girls at ages 7 9 years, but cord blood PCB levels were unrelated to BMI in either boys nor girls. Maternal PCB levels in pregnancy of Faroese women were related to higher WC in female children at aged 7 years who had overweight mothers [48]. Additionally, these authors found a significant association between prenatal PCBs and a change of BMI in girls from 5 to 7 years of age as well as BMI at 7 years [48]. In the INMA Spanish cohort study, the concentration of PCBs in cord blood positively predicted BMI and overweight in Spanish children aged 6.5 years, in contrast to NS findings at earlier ages, an association that appeared to be stronger in girls than in boys [38]. A pooled analysis of seven European birth cohorts [49•], the largest study to date examining obesogenic effects of POPs, found a negative association between postnatal exposure to PCBs and weight gain from birth to 24 months. Nevertheless, the heterogeneity of levels of DDE exposure introduced by pooling cohorts potentially could lead to imprecise estimates of these associations.

\section{Organochlorine Pesticides}

Some persistent organochlorine pesticides have been suspected to play a prominent role in the development of early-onset obesity. Of the 12 prospective studies of maternal exposure to DDE or DDT, 8 found a significant positive association with obesity in offspring (Table 1). Among maternal-child dyads in the Spanish INMA cohort, in utero DDE exposure was related to rapid growth in the first 6 months of life and subsequent overweight at age 14 months [42•, 43]. In the same cohort, DDE concentrations in cord blood were also related to an increased risk of overweight at 6.5 years of age but DDT was associated with overweight in boys [38]. Across seven pooled European birth cohorts, DDE levels in cord blood were associated with greater weight change from birth to 24 months of age, but this effect was not seen in infants with postnatal exposure via breastfeeding [49•]. Studies [47, 48] conducted in Flemish and in Faroese children reported significant positive associations between prenatal DDE and BMI change from 5 to 7 years of age and obesity at 7 years in girls but not in boys, respectively. Two reports from the Center for the Health Assessment 
of Mothers and Children of Salinas (CHAMACOS) study, however, found that prenatal DDT concentrations were related to increased BMI and WC at 9 years of age, but only in boys [50]. These authors also documented a significant trend of increased odds of obesity across ages $2,3.5,5$, and 7 years in boys and girls combined [51].

Despite a preponderance of evidence linking persistent organochlorine pesticides to offspring weight gain and status in US and European settings, four recent studies found no association of developmental exposure to DDE with infant or child obesity (Table 1). For instance, one study of 253 women residing in the state of Morelos, Mexico, suggested that maternal exposure to DDE during pregnancy may not affect infant growth [52]. Similarly, three prospective studies found no clear association between maternal exposure to DDE or DDT and growth in the first year of age of children in Netherlands [44•] or BMI in Greenlandic, Polish, and Ukrainian children aged 5 to 9 years [45] or 7-year-old children in USA with relatively high DDE exposure in utero [46], respectively.

Few human studies have examined early life exposures to $\mathrm{HCB}$ and $\mathrm{HCH}$ (Table 1). Of four epidemiological studies considering these perinatal exposures, three found no significant association between prenatal HCB and infant growth at 6 and 14 months [42 ${ }^{\bullet}$, obesity in children aged 7 years [46], or obesity in children aged 7 to 9 years old [47], respectively. No association with $\mathrm{HCH}$ was found in these three studies. Only one study reported HCB was positively associated with rapid growth and overweight in Spanish infants in the INMA cohort [43].

\section{Other POPs}

Other new POPs on the Stockholm Convention list, including PFOA, PFOS, PBDEs, and TBT, have attracted attention because their potential to promote obesity has been suggested by several animal studies. We identified only five longitudinal studies since 2011 that evaluated the effects of PFOA and PFOS on offspring obesity in humans (Table 1). British girls at 20 months of age with prenatal PFOS exposure in the upper tertile were $580 \mathrm{~g}$ heavier comparing with those in the lower tertile, but no differences in weight were found with PFOA [53]. In contrast, a Danish cohort study [54•] of 665 pregnant women found that in utero exposure to PFOA was associated with increased overweight and WC among female offspring followed to early adulthood, as well as levels of insulin, adiponectin, and leptin, but no association was observed for PFOS [54•]. One prospective study observed a null association of prenatal PFOA/PFOS with infant BMI at 24 months [44-], and two found no significant association with overweight in school-aged children $[55,56]$. In the single human study of developmental exposure to PBDEs and risk of obesity in the Salinas Valley, California, maternal PBDE serum levels during pregnancy were significantly related to decreased BMI in 7-year-old girls [57]. Despite the relatively extensive number of experimental studies of TBT, we identified only one study exploring the possible obesogenic effect of TBT in humans. A Finnish cohort study presents that TBT levels in placental tissue were associated with infant weight gain during the first 3 months of life, but no associations were observed at 3 or 18 months [58].

\section{Short-lived Ubiquitous Pollutants}

\section{Bisphenol A}

Bisphenol A (BPA) is used in a wide range of consumer products including can linings and packaging materials and in children's toys. BPA is considered an EDC that can regulate insulin and leptin production by exerting estrogenic activity, acting as agonist and antagonist of PPAR $\gamma$ [27]. Experimental data suggest that prenatal BPA promotes weight gain in offspring; but this association has not extensively been studied in humans in longitudinal studies. Three prospective studies that examined the effects of urinary BPA levels during pregnancy on postnatal growth and obesity report inconsistent results [59-61]. In the Spanish population participating in the INMA study, prenatal BPA was weakly associated with increased WC in 4-year-old children but not at earlier ages [59]. In the CHAMACOS cohort, maternal urinary BPA concentrations were inversely associated with BMI and obesity in 9-year-old Mexican-American girls, effects that were not yet evident at 5 years [60]. In the same population, BPA exposure during late pregnancy was related to increased leptin in boys whereas BPA in early pregnancy was associated with increased adiponectin in girls at 9 years of age [61]. Although the mechanistic pathways for sex-specific effects seen in these studies are not fully understood, one potential explanation is that BPA may affect estrogen activity by interrupting original binding at nuclear estrogen receptors. The synthesis and function of estrogen as well as the distribution of estrogen receptors vary in males and females [62].

\section{Phthalates}

Phthalates (monoethylhexyl phthalate (MEHP), diethylhexyl phthalate (DEHP), and oxidative DEHP metabolite mono-(2ethyl-5-oxohexyl) phthalate (MEOHP)) are used in hundreds of consumer products including plastics, cosmetics, and personal care products. Growing experimental evidence has shown that phthalates are thyroid hormone and androgen antagonists and may affect adipogenesis, lipid accumulation, and insulin resistance by regulating activation of PPAR $\gamma$ [63]. Previous studies of associations between prenatal phthalate exposure and obesity among children are limited to a few cross-sectional reports. A recent prospective study in a small Danish sample $(n=61)$ reported that higher MEOHP in cord blood was related to lower BMI in male offspring from birth to 
11 months old, whereas a reverse association was revealed in female children [44•]. Similar results were seen in a prospective study of DEHP and postnatal growth in the Spanish INMA-Sabadell Birth Cohort; prenatal exposure to DEHP was associated with reduced weight gain in the first 6 months of life and lower BMI at 4 to 7 years in boys [64].

\section{Conclusions}

The 22 studies discussed here reveal inconsistent conclusions about obesogenic effects of maternal exposure to man-made compounds that may be attributable to differences in the study population, chemical congeners, levels of exposure, time windows for outcomes, and measured and unmeasured confounders. To date, the mechanistic pathways to explain such associations are not fully understood. Most of human studies on the effects of early-life exposure to synthetic chemicals on overweight or obesity in later life have focused on the prenatal period, whereas few have examined exposure via breastfeeding or combination of both. Rapid growth, overweight, and obesity among children were largely assessed using indirect measures of adiposity including weight gain, $\mathrm{BMI}$ and $\mathrm{WC}$, and direct measures were less commonly employed, e.g., skin folds, bio-impedance, dual-energy Xray absorptiometry, or adipokines. Most current epidemiological data consider the influence of these chemicals on overweight and obesity during infancy and early childhood, up to 9 years of age, but few studies examine the persistence of obesity in adolescence and adulthood due to challenges of long-term follow-up. Of the reports we reviewed, the majority focused on understanding the obesogenicity of DDE and PCBs, while investigations of other environmental toxicants were scarce, particularly for PBDEs, organotin compounds and nonpersistent chemicals. Overall, we found continued support for a predominantly positive effect for maternal DDE and child obesity and less consistent associations for other substances. Several studies were limited by relatively small to modest sample sizes, whereas those with large sample size were constrained by heterogeneity across pooled populations which could contribute to imprecision. Many authors modeled relationships with categorical measures of exposure, typically quartiles, which may reduce statistical power. Given that quantiles of exposure were not necessarily assigned according to clinical relevance, these studies also might not reveal biologically meaningful associations. Nevertheless, categorical measures could reveal nonlinear or non-monotonic relationships that could be explored in studies with larger sample sizes. A strength of the 22 studies reviewed here was that all utilized a prospective, longitudinal design that can enhance causal inference. A few studies used advanced modeling for estimating postnatal exposure and measured levels of adipokines and skinfolds to assess adiposity.
Further research in larger study populations and various settings worldwide is needed to confirm the observed associations, especially for less studied chemicals, and to evaluate potential underlying mechanisms. Studies with longer follow-up time to ascertain the persistence of compounds' effects into later ages and developmental periods, including the transition to young adulthood, should be considered as a priority for future investigations. Research with complete information about both maternal prenatal and postnatal exposure and that utilize direct measures of adiposity is recommended. Considering that many studies report inconsistent results regarding differential effects on boys and girls, the systematic evaluation of interaction by sex is needed. Other factors that appear to serve as effect modifiers of chemical exposures, such as intake of fat, maternal weight status, and birth weight, also require confirmation in larger studies. Among these, birth weight deserves special attention as it shows divergent functions in different analysis. For instance, birth weight has been considered a confounder that may compound the effect on obesity and alternatively has been treated as a variable in the causal pathway. Lastly, studies of mixtures of chemicals should be taken into consideration given that humans have detectable levels of various obesogenic compounds.

In conclusion, the studies of risk of obesity in relation to environmental obesogen exposures during early life provide suggestive evidence for some but not all chemicals, and many uncertainties require further exploration. With prospective study designs, large sample size, improved exposure assessment, direct measures of obesity, and advanced statistical analysis, data generated from these studies can contribute to a strong evidence base for recommendations and strategies to prevent pediatric obesity and its long-term sequelae.

Acknowledgments This work was supported in part by 1P01ES022844-01/RD-83543601.

\section{Compliance with Ethics Guidelines}

Conflict of Interest The authors declare that they have no competing interests.

Human and Animal Rights and Informed Consent This article does not contain any studies with human or animal subjects performed by any of the authors.

\section{References}

Papers of particular interest, published recently, have been highlighted as:

- Of importance

1. World Health Organization. Childhood overweight and obesity. 2014; Available from: http://www.who.int/end-childhood-obesity/ facts/en/. 
2. Ogden CL, Carroll MD, Kit BK, et al. Prevalence of childhood and adult obesity in the United States, 2011-2012. JAMA. 2014;311: 806-14.

3. National Center for Health Statistics. Health, United States, 2013: with special feature on prescription drugs. Hyattsville: National Center for Health Statistics (US); 2014.

4. Freedman DS, Mei Z, Srinivasan SR, et al. Cardiovascular risk factors and excess adiposity among overweight children and adolescents: the Bogalusa Heart Study. J Pediatr. 2007;150:12-7. e2.

5. Li C, Ford ES, Zhao G, et al. Prevalence of pre-diabetes and its association with clustering of cardiometabolic risk factors and hyperinsulinemia among U.S. adolescents: National Health and Nutrition Examination Survey 2005-2006. Diabetes Care. 2009;32:342-7.

6. Daniels SR, Arnett DK, Eckel RH, et al. Overweight in children and adolescents: pathophysiology, consequences, prevention, and treatment. Circulation. 2005;111:1999-2012.

7. Dietz WH. Overweight in childhood and adolescence. N Engl J Med. 2004;350:855-7.

8. U.S. Department of Health and Human Services. The Surgeon General's call to action to prevent and decrease overweight and obesity. Rockville: U.S. Department of Health and Human Services, Public Health Service, Office of the Surgeon General; 2001

9. Maffeis C, Tato L. Long-term effects of childhood obesity on morbidity and mortality. Horm Res. 2001;55 Suppl 1:42-5.

10. Must A, Jacques PF, Dallal GE, et al. Long-term morbidity and mortality of overweight adolescents: a follow-up of the Harvard growth study of 1922 to 1935 . N Engl J Med. 1992;327:1350-5.

11. Hoffmans MD, Kromhout D, et al. The impact of body mass index of 78,612 18-year old Dutch men on 32-year mortality from all causes. J Clin Epidemiol. 1988;41:749-56.

12. Srinivasan SR, Bao W, Wattigney WA, et al. Adolescent overweight is associated with adult overweight and related multiple cardiovascular risk factors: the Bogalusa heart study. Metabolism. 1996;45: 235-40.

13. Office of the Surgeon General (US). The Surgeon General's vision for a healthy and fit nation. Rockville: Office of the Surgeon General (US); 2010.

14. Cawley J, Meyerhoefer C. The medical care costs of obesity: an instrumental variables approach. National Bureau of Economic Research. Cambridge 2010. Available from: http://www.nber.org/ papers/w16467.

15. Wang Y, Beydoun MA, Liang L, et al. Will all Americans become overweight or obese? Estimating the progression and cost of the US obesity epidemic. Obesity. 2008;16:2323-30.

16. Singh AS, Mulder C, Twisk JW, et al. Tracking of childhood overweight into adulthood: a systematic review of the literature. Obes Rev. 2008;9:474-88.

17. Baillie-Hamilton PF. Chemical toxins: a hypothesis to explain the global obesity epidemic. J Altern Complement Med. 2002;8:18592.

18. Grun F, Blumberg B. Environmental obesogens: organotins and endocrine disruption via nuclear receptor signaling. Endocrinology. 2006;147:S50-5.

19. Landrigan PJ, Goldman LR. Children's vulnerability to toxic chemicals: a challenge and opportunity to strengthen health and environmental policy. Health Aff. 2011;30:842-50.

20. Grun F, Blumberg B. Endocrine disrupters as obesogens. Mol Cell Endocrinol. 2009;304:19-29.

21. Blumberg B, Iguchi T, Odermatt A. Endocrine disrupting chemicals. J Steroid Biochem Mol Biol. 2011;127:1-3.

22. Grun F, Blumberg B. Minireview: the case for obesogens. Mol Endocrinol. 2009;23:1127-34.

23. Desai M, Beall M, Ross MG. Developmental origins of obesity: programmed adipogenesis. Curr Diab Rep. 2013;13:27-33.
24. Kelishadi R, Poursafa P, Jamshidi F. Role of environmental chemicals in obesity: a systematic review on the current evidence. J Environ Public Health. 2013;2013:896789.

25. Janesick A, Blumberg B. Endocrine disrupting chemicals and the developmental programming of adipogenesis and obesity. Birth Defects Res C Embryo Today. 2011;93:34-50.

26. La Merrill M, Birnbaum LS. Childhood obesity and environmental chemicals. Mt Sinai J Med. 2011;78:22-48.

27. Grant KLCD, Sly LJ, Sly PD. Environmental contributions to obesity and type 2 diabetes. J Environ Immunol Toxicol. 2014;1:80 91.

28. Tang-Peronard JL, Andersen HR, Jensen TK, et al. Endocrinedisrupting chemicals and obesity development in humans: a review. Obes Rev. 2011;12:622-36.

29. Arsenescu V, Arsenescu RI, King V, et al. Polychlorinated biphenyl-77 induces adipocyte differentiation and proinflammatory adipokines and promotes obesity and atherosclerosis. Environ Health Perspect. 2008;116:761-8.

30. Adigun AA, Wrench N, Seidler FJ, et al. Neonatal organophosphorus pesticide exposure alters the developmental trajectory of cell-signaling cascades controlling metabolism: differential effects of diazinon and parathion. Environ Health Perspect. 2010;118:210-5.

31. Adigun AA, Seidler FJ, Slotkin TA. Disparate developmental neurotoxicants converge on the cyclic AMP signaling cascade, revealed by transcriptional profiles in vitro and in vivo. Brain Res. 2010;1316:1-16.

32. Vested A, Giwercman A, Bonde JP, et al. Persistent organic pollutants and male reproductive health. Asian J Androl. 2014;16:71-80.

33. Yu GW, Laseter J, Mylander C. Persistent organic pollutants in serum and several different fat compartments in humans. J Environ Public Health. 2011;2011:417980.

34. Li QQ, Loganath A, Chong YS, et al. Persistent organic pollutants and adverse health effects in humans. J Toxic Environ Health A. 2006;69:1987-2005.

35. Woodruff TJ, Zota AR, Schwartz JM. Environmental chemicals in pregnant women in the United States: NHANES 2003-2004. Environ Health Perspect. 2011;119:878-85.

36. Foster W, Chan S, Platt L, et al. Detection of endocrine disrupting chemicals in samples of second trimester human amniotic fluid. J Clin Endocrinol Metab. 2000;85:2954-7.

37. Longnecker MP, Rogan WJ, Lucier G. The human health effects of DDT (dichlorodiphenyltrichloroethane) and PCBS (polychlorinated biphenyls) and an overview of organochlorines in public health. Annu Rev Public Health. 1997;18:211-44.

38. Valvi D, Mendez MA, Martinez D, et al. Prenatal concentrations of polychlorinated biphenyls, DDE, and DDT and overweight in children: a prospective birth cohort study. Environ Health Perspect. 2012;120:451-7.

39. Ahlborg UG, Brouwer A, Fingerhut MA, et al. Impact of polychlorinated dibenzo-p-dioxins, dibenzofurans, and biphenyls on human and environmental health, with special emphasis on application of the toxic equivalency factor concept. Eur J Pharmacol. 1992;228:179-99.

40. Casals-Casas C, Feige JN, Desvergne B. Interference of pollutants with PPARs: endocrine disruption meets metabolism. Int J Obes. 2008;32 Suppl 6:S53-61.

41. Cooke PS, Naaz A. Role of estrogens in adipocyte development and function. Exp Biol Med. 2004;229:1127-35.

42. Mendez MA, Garcia-Esteban R, Guxens M, et al. Prenatal organochlorine compound exposure, rapid weight gain, and overweight in infancy. Environ Health Perspect. 2011;119:272-8. The author of this paper is the first to report that prenatal DDE exposure during pregnancy was linked to rapid growth starting as early as 6 months of life. 
43. Valvi D, Mendez MA, Garcia-Esteban R, et al. Prenatal exposure to persistent organic pollutants and rapid weight gain and overweight in infancy. Obesity. 2014;22:488-96.

44. de Cock M, de Boer MR, Lamoree M, et al. First year growth in relation to prenatal exposure to endocrine disruptors - a Dutch prospective cohort study. Int J Environ Res Public Health. 2014;11: 7001-21. This is the first study of postnatal growth in early childhood in relation to prenatal phthalate exposure measured in cord blood

45. Hoyer BB, Ramlau-Hansen CH, Henriksen TB, et al. Body mass index in young school-age children in relation to organochlorine compounds in early life: a prospective study. Int J Obes. 2014;38: 919-25.

46. Cupul-Uicab LA, Klebanoff MA, Brock JW, et al. Prenatal exposure to persistent organochlorines and childhood obesity in the US collaborative perinatal project. Environ Health Perspect. 2013;121: 1103-9.

47. Delvaux I, Van Cauwenberghe J, Den Hond E, et al. Prenatal exposure to environmental contaminants and body composition at age 7-9 years. Environ Res. 2014;132:24-32.

48. Tang-Peronard JL, Heitmann BL, Andersen HR, et al. Association between prenatal polychlorinated biphenyl exposure and obesity development at ages 5 and $7 \mathrm{y}$ : A prospective cohort study of 656 children from the Faroe islands. Am J Clin Nutr. 2014;99:5-13.

49. Iszatt N, Stigum H, Verner MA, et al. Prenatal and postnatal exposure to persistent organic pollutants and infant growth: a pooled analysis of seven European birth cohorts. Environ Health Perspect. 2015;123:730-6. This is the largest study to date examining associations between POPs exposure and postnatal growth during infancy featured with an advanced modeling method to estimate maternal exposure.

50. Warner M, Wesselink A, Harley KG, et al. Prenatal exposure to dichlorodiphenyltrichloroethane and obesity at 9 years of age in the CHAMACOS study cohort. Am J Epidemiol. 2014;179: 1312-22.

51. Warner M, Schall R, Harley KG, et al. In utero DDT and DDE exposure and obesity status of 7-year-old Mexican-American children in the CHAMACOS cohort. Environ Health Perspect. 2013;121:631-6.

52. Garced S, Torres-Sanchez L, Cebrian ME, et al. Prenatal dichlorodiphenyldichloroethylene (DDE) exposure and child growth during the first year of life. Environ Res. 2012;113:58-62.

53. Maisonet M, Terrell ML, McGeehin MA, et al. Maternal concentrations of polyfluoroalkyl compounds during pregnancy and fetal and postnatal growth in British girls. Environ Health Perspect. 2012;120:1432-7.

54. Halldorsson TI, Rytter D, Haug LS, et al. Prenatal exposure to perfluorooctanoate and risk of overweight at 20 years of age: a prospective cohort study. Environ Health Perspect. 2012;120:668

73. This is the study with the longest follow-up time until young adulthood and strengthened by using biomarkers of adiposity to assess outcomes.

55. Andersen CS, Fei C, Gamborg M, et al. Prenatal exposures to perfluorinated chemicals and anthropometry at 7 years of age. Am J Epidemiol. 2013;178:921-7.

56. Hoyer BB, Ramlau-Hansen CH, Vrijheid M, et al. Anthropometry in 5- to 9-year-old Greenlandic and Ukrainian children in relation to prenatal exposure to perfluorinated alkyl substances. Environ Health Perspect. 2015;123:841-6.

57. Erkin-Cakmak A, Harley KG, Chevrier J, et al. Childhood polybrominated diphenyl ether exposures and body mass at age 7 years: the CHAMACOS study. Environ Health Perspect. 2015;123: 636-42.

58. Rantakokko P, Main KM, Wohlfart-Veje C, et al. Association of placenta organotin concentrations with growth and ponderal index in 110 newborn boys from Finland during the first 18 months of life: a cohort study. Environ Heal. 2014;13:45.

59. Valvi D, Casas M, Mendez MA, et al. Prenatal bisphenol A urine concentrations and early rapid growth and overweight risk in the offspring. Epidemiology. 2013;24:791-9.

60. Harley KG, Schall R, Chevrier J, et al. Prenatal and postnatal bisphenol a exposure and body mass index in childhood in the CHAMACOS cohort. Environ Health Perspect. 2013;121:514-20.

61. Volberg V, Harley K, Calafat AM, et al. Maternal bisphenol a exposure during pregnancy and its association with adipokines in Mexican-American children. Environ Mol Mutagen. 2013;54: $621-8$.

62. Gillies GE, McArthur S. Estrogen actions in the brain and the basis for differential action in men and women: a case for sex-specific medicines. Pharmacol Rev. 2010;62:155-98.

63. Hao C, Cheng X, Xia H, et al. The endocrine disruptor mono-(2ethylhexyl) phthalate promotes adipocyte differentiation and induces obesity in mice. Biosci Rep. 2012;32:619-29.

64. Valvi D, Casas M, Romaguera D, et al. Prenatal phthalate exposure and childhood growth and blood pressure: evidence from the Spanish INMA-Sabadell birth cohort study. Environ Health Perspect. 2015. doi:10.1289/ehp.1408887. 